\title{
Creating a Universal Data Release Policy Across Programs in a State Health Department
}

\author{
Ekaette Joseph-Isang* \\ Kentucky Department for Public Health, Frankfort, KY, USA
}

\section{Objective}

To describe the process of producing a universal data release policy for use by different programs in a state health department

\section{Introduction}

The introduction of electronic health systems has led to easier collation, compilation, and analysis of data as well as easier access. For data to be put to be impactful use, it must be shared both for research and decision making purposes. Data sharing and release should neither compromise privacy nor lead to wrong conclusions. The need to share information that guides policies and decision making should be balanced with the need for the data to be reliable. The aim was to produce a data release policy to be used as a baseline tool to guide the practice of data release and sharing across programs and with outside requesters.

\section{Methods}

Data release polices take into consideration different parameters as determined by the sponsoring organization. Data release policies were collected from different programs within and outside the state department of health. Spreadsheets and cohort brainstorming session were employed. Parameters, suppression rules and cautionary release rules were tabulated and analyzed on spreadsheets to compare similarities and differences. A data release work group was constituted. Preliminary findings showed that most data release policies were based on a variety of parameters. Most used a combination of parameters

Parameters included the total population, number of count, relative standard errors, confidence intervals and in some cases geographical areas. Program areas were consulted with regard their preferred parameters.

Program policies were also compared with those available on national data sites e.g. NCHS and CDC wonder. Survey data and data from counts were treated using different parameters with the aim of preserving confidentiality and endure reliability. Based on the most frequent data requests, parameters that guaranteed confidentiality and reliability were agreed upon by the work group. This report outlines the process for creating a universally acceptable data release policy for the state health department and the advantages and disadvantages of using certain parameters.

\section{Results}

A universal data release policy acceptable to different programs was produced with allowances for tightening the protocol where specific programs required more stringent data release policies.

\section{Conclusions}

Data release policies make for ease of handling data requests. There is no one size fits all. Having a data release policy allows program managers to have a reference point for evaluating data requests. A data release algorithm provides an easily comprehensible visual tool. Programs can be more stringent as their program peculiarities dictate but the data release policy serves as the minimum necessary to guide the process.

\section{Keywords}

data release; data sharing; state health department; data policy; data sharing agreement

*Ekaette Joseph-Isang

E-mail: ejosephisang@gmail.com 